ORIGINAL ARTICLE

\title{
Do physical leisure time activities prevent fatigue? A 15 month prospective study of nurses' aides
}

\author{
W Eriksen, D Bruusgaard
}

Br J Sports Med 2004;38:331-336. doi: 10.1136/bjsm.2002.004390

See end of article for authors' affiliations .....................

Correspondence to: Dr Eriksen, General Practice and Community Medicine, University of Oslo, PO Box 1130 , Blindern, Oslo 0318, Norway; w.b.eriksen@ samfunnsmed.vio.no

Accepted 20 May 2003

\begin{abstract}
Objective: To test the hypothesis that physical leisure time activities reduce the risk of developing persistent fatigue.

Methods: The hypothesis was tested in a sample that was homogeneous with respect to sex and occupation, with a prospective cohort design. Of 6234 vocationally active, female, Norwegian nurses' aides, not on leave because of illness or pregnancy when they completed a mailed questionnaire in 1999, $5341(85.7 \%)$ completed a second questionnaire 15 months later. The main outcome measure was the prevalence of persistent fatigue-that is, always or usually feeling fatigued in the daytime during the preceding 14 days.

Results: In participants without persistent fatigue at baseline, reported engagement in physical leisure time activities for 20 minutes or more at least once a week during the three months before baseline was associated with a reduced risk of persistent fatigue at the follow up lodds ratio $=0.70 ; 95 \%$ confidence interval 0.55 to 0.89 ), after adjustments for age, affective symptoms, sleeping problems, musculoskeletal pain, long term health problems of any kind, smoking, marital status, tasks of a caring nature during leisure time, and work factors at baseline.

Conclusion: The study supports the hypothesis that physical leisure time activities reduce the risk of developing persistent fatigue.
\end{abstract}

$\mathrm{P}$ ersistent fatigue is a common complaint, with a prevalence of $15-20 \%$ in the general population. ${ }^{12}$ It may have a strong negative effect on functional ability and quality of life. ${ }^{3}$ In a study of Norwegian nurses' aides, the $15 \%$ with persistent fatigue had a $73 \%$ increased risk of becoming sick listed during the next three months, after adjustments for affective symptoms, sleeping problems, musculoskeletal pain, demographic characteristics, tasks of a caring nature during leisure time, and work factors. ${ }^{4}$

Persistent fatigue is a non-specific symptom, which may accompany physical diseases, psychiatric disorders, sleep disturbance, and pregnancy. ${ }^{15-9}$ It has also been associated with psychosocial stress at work and in private life. ${ }^{6}{ }^{10}$ The biological basis of persistent fatigue is often unclear, even when it appears in connection with clear cut somatic diseases such as cancer. ${ }^{11}$

Cross sectional studies have shown an association between physical inactivity and the prevalence of persistent fatigue. ${ }^{16}{ }^{12}$ In a national probability sample of 25-74 year olds in the United States, those who were physically inactive were more than twice as likely to report frequently feeling tired or worn out as those who were physically active. ${ }^{1}$ In 4318 male Israeli industrial employees, those who participated in physical activity less than once a week had a 1.7-fold higher prevalence of "frequent fatigue" than those who engaged in physical activity at least once a week. ${ }^{12}$ Of 123 women from Toronto, Canada who suffered from fatigue, one third attributed their fatigue to lack of exercise. ${ }^{6}$ However, the cross sectional design of these studies makes it difficult to say whether the associations are due to effects of exercise or to the fact that fatigued subjects may be less inclined to participate in physical activities. In the only prospective epidemiological study in this field, a study of a mixed working population in the Netherlands, Bültman and coworkers ${ }^{13}$ found that low physical activity during leisure time was a predictor of fatigue, but only in men. Recent experimental studies have shown that physical exercise under certain circumstances may have a positive effect on people's perceived level of vigour and energy. ${ }^{14-16}$ In a repeated measures design, Hansen et $a l^{14}$ found that 10 minutes of ergometer cycling with moderate effort had an immediate and positive effect on the level of vigour. In two clinical trials, ${ }^{15}{ }^{16}$ one of which was randomised, ${ }^{16}$ Dimeo et al found that cancer patients who had carried out an endurance training programme in association with high dose chemotherapy and peripheral blood stem cell transplantation were less likely to report feeling fatigue with usual daily activities ${ }^{15}$ and less likely to report increased fatigue scores ${ }^{16}$ than patients who did not train.

The aim of this study was to test the hypothesis that physical leisure time activities reduce the risk of developing persistent fatigue.

\section{METHODS}

Design

The study had a prospective cohort design.

\section{Subjects}

The Norwegian Union of Health and Social Workers organises the most of the certified nurses' aides in Norway. In 1999, a random sample organised by this union were mailed a questionnaire. After one reminder, 7478 of 12000 $(62.3 \%)$ completed and returned the questionnaire. The sample of this study comprised the 6234 vocationally active, female respondents who were not on leave because of illness or pregnancy. Of these, $5341(85.7 \%)$ completed a second questionnaire 15 months later.

\section{Outcome measure}

At baseline and follow up, the respondents were asked: "During the previous 14 days, how did you feel during the day?". There was a check list with the following optional 
answers: "always fit"; "usually fit"; "varied between fit and fatigued"; "usually fatigued"; "always fatigued". Persistent fatigue was operationally defined as reporting "usually fatigued" or "always fatigued". The outcome measure was the prevalence of persistent fatigue.

\section{Independent variables}

At baseline, demographic characteristics, physical leisure time activities, smoking, tasks of a caring nature during leisure time, work factors, and health complaints were recorded, as described in detail elsewhere. ${ }^{17}$

The question about physical leisure time activities was worded as follows: "During the previous three months, what kind of physical exercise have you done regularly in your leisure time? By regularly, we mean 20 minutes or more at least once a week." There was a check list with the following optional answers: "slow walks"; "brisk walks"; "jogging or running"; "aerobics or gymnastics"; "dancing"; "swimming"; "other"; "no regular exercise".

Sleeping problems were measured with the following question in the basic nordic sleep questionnaire ${ }^{18}$ : "How well have you been sleeping during the past three months?". Optional answers were: "well"; "rather well"; "neither well nor badly"; "rather badly"; "badly".

Affective symptoms during the preceding 14 days were measured with five questions in the symptom check list (SCL): a sum of these questions correlated in a former study at $r=0.92$ with the global SCL-25 score. ${ }^{19}$ In the present study, the sum was divided into quintiles.

Musculoskeletal pain during the preceding 14 days was recorded using a questionnaire that was a modification of the standardised nordic questionnaire. ${ }^{20}$ There were questions about the intensity of pain in the head, neck, shoulder, elbow, hand or wrist, upper back, low back, hip, knee, and ankle or foot. There was a check list for each part of the body with the following optional answers: "not bothered"; "a little bothered"; "rather intensely bothered"; "very intensely bothered". The questions were supplemented with a diagram showing regions of pain. The intensity of musculoskeletal pain was defined as the intensity in the most painful part of the body. Widespread pain was defined as pain in the midline area, upper limbs, and lower limbs.

The respondents were also asked to report the extent to which they had been bothered by low back pain and neck pain during the preceding three months.

Long term health problems were ascertained from the question: "Do you have any kind of long term or chronic health problem (for instance, asthma, arthritis, chronic pain)?". Optional answers were: "yes but it does not bother me"; "yes and it bothers me to some extent"; "yes and it bothers me a lot"; "no".

Work factors were measured with the standardised questionnaire QPSNordic. ${ }^{21}$

\section{Ethics}

The research protocol was approved by the committee for medical research ethics. Informed written consent was given by the responders.

\section{Statistical analysis}

Statistical analyses were conducted with the Statistical Package for Social Sciences (SPSS) version 6.1. $\chi^{2}$ tests and logistic regression analyses were used.

Logistic regression analyses were performed to adjust for potential confounders of the relation between physical leisure time activities and the outcome measure. Two series of logistic regression analyses were conducted. In the first series, no regular activity was compared with regular physical leisure time activity of any kind, and in the second series, it was compared with specific activities. To ensure informative comparisons with satisfactory statistical power, a variable with the following five mutually exclusive categories was composed: no regular physical leisure activity; slow walks (as the only activity); brisk walks (as the only activity or in combination with slow walks); aerobics or gymnastics (as the only activity or in combination with slow walks); other activities or combinations of activities. Subjects who had reported jogging or running, swimming, or dancing were included in the last and mixed category together with those who had marked the alternative "other kind of regular activity", because few engaged in these activities and there was considerable overlap between the activities.

Three different logistic regression models were tested. In the first model, adjustments were made for age. In the second one, adjustments were made for age and health complaints at baseline-that is, level of sleeping problems in preceding three months, intensity of low back pain and neck pain in preceding three months, intensity of musculoskeletal pain of any kind in preceding 14 days, presence of widespread pain in preceding 14 days, level of affective symptoms in preceding 14 days, level of fatigue/fitness in preceding 14 days, and the extent to which the respondents were bothered by long term or chronic health problems of any kind. In the third model, adjustments were made for age, baseline health complaints, marital status, number of preschool children, the extent to which the subjects were engaged in special tasks of a caring nature in their leisure time-for example, taking care of handicapped child or elderly relatives-daily consumption of cigarettes, former smoking, number of working hours a week, frequency of night shifts, quantitative work demands, the extent to which the work required physical endurance, positive challenges in the job, the extent to which the culture in the work unit was supportive and encouraging, and level of personal engagement in the work unit. All factors were entered in the models simultaneously.

The Hosmer-Lemeshow test, overall rate of correct classification, and area under the receiver operating characteristic (ROC) curve were used to assess the fit of the models. The area under the ROC curve was estimated with the help of the Mann-Whitney $U$ statistic, as described by Hosmer and Lemeshow. ${ }^{22}$

\section{RESULTS}

\section{Characteristics of respondents and dropouts}

Table 1 shows baseline characteristics of those who completed both questionnaires (hereafter referred to as respondents) and those who dropped out between baseline and the follow up (dropouts). The respondents were older, more often married or cohabiting, working fewer hours a week, more often non-smokers, and less bothered by affective symptoms than the dropouts.

\section{Physical leisure time activities and prevalence of persistent fatigue at baseline}

Table 2 shows the relation between reported engagement in physical leisure time activities during the three months before baseline and the prevalence of persistent fatigue at baseline. Respondents who reported engagement in physical leisure time activities had lower prevalence of persistent fatigue than those who did not report such activities. This association between reported physical activity and prevalence of fatigue was significant when all the different activity categories were compared in an overall $\chi^{2}$ test $(p=0.000)$ and when any regular activity - that is, all types of regular activity combined in one category-was compared with no regular activity in a $\chi^{2}$ test $(\mathrm{p}=0.000)$. 
Table 1 Baseline characteristics of the respondents and dropouts

\begin{tabular}{|c|c|c|c|c|}
\hline \multirow[b]{2}{*}{ Characteristics at baseline } & \multicolumn{2}{|c|}{ Respondents ( $N=5341)$} & \multicolumn{2}{|c|}{ Dropouts ( $\mathrm{N}=893$ ) } \\
\hline & $n$ & $\%$ & $n$ & $\%$ \\
\hline \multicolumn{5}{|l|}{ Age } \\
\hline$<30$ & 337 & 6.3 & 151 & 16.9 \\
\hline $30-39$ & 1029 & 19.3 & 208 & 23.3 \\
\hline $40-49$ & 2203 & 41.3 & 314 & 35.2 \\
\hline $50-59$ & 1538 & 28.8 & 177 & 19.8 \\
\hline$>59$ & 233 & 4.4 & 42 & 4.7 \\
\hline \multicolumn{5}{|l|}{ Marital status } \\
\hline Married or cohabiting & 4358 & 81.7 & 684 & 76.9 \\
\hline Single & 975 & 18.3 & 206 & 23.1 \\
\hline \multicolumn{5}{|l|}{ Hours worked a week } \\
\hline $1-18$ & 708 & 13.4 & 107 & 12.1 \\
\hline $19-36$ & 3945 & 74.9 & 646 & 73.3 \\
\hline$>36$ & 613 & 11.6 & 128 & 14.5 \\
\hline \multicolumn{5}{|l|}{ Cigarettes smoked a day } \\
\hline 0 & 3059 & 58.4 & 453 & 51.9 \\
\hline $1-9$ & 1198 & 22.9 & 223 & 25.5 \\
\hline $10-19$ & 894 & 17.1 & 182 & 20.8 \\
\hline 20 or more & 88 & 1.7 & 15 & 1.7 \\
\hline \multicolumn{5}{|l|}{ Physical leisure activity } \\
\hline No regular exercise & 1359 & 25.4 & 230 & 25.8 \\
\hline Slow walks* & 1009 & 18.9 & 177 & 19.8 \\
\hline Brisk walkst & 1129 & 21.1 & 165 & 18.5 \\
\hline Aerobics or gymnastics $\dagger$ & 312 & 5.8 & 58 & 6.5 \\
\hline Other activities or combinations & 1532 & 28.7 & 263 & 29.5 \\
\hline \multicolumn{5}{|l|}{ Fatigue } \\
\hline Always fit & 169 & 3.2 & 22 & 2.5 \\
\hline Usually fit & 1658 & 31.1 & 252 & 28.3 \\
\hline Varied between fit and fatigued & 2698 & 50.7 & 472 & 52.9 \\
\hline Usually fatigued & 710 & 13.3 & 133 & 14.9 \\
\hline Always fatigued & 88 & 1.7 & 13 & 1.5 \\
\hline \multicolumn{5}{|l|}{ Long term health problems } \\
\hline No & 3432 & 65.0 & 593 & 67.0 \\
\hline Yes, but not bothered & 393 & 7.4 & 74 & 8.4 \\
\hline Yes, it bothers me some & 1048 & 19.8 & 167 & 18.9 \\
\hline Yes, it bothers me a lot & 410 & 7.8 & 51 & 5.8 \\
\hline \multicolumn{5}{|l|}{ Affective symptoms } \\
\hline 1 and 2 & 2329 & 44.0 & 342 & 39.0 \\
\hline 3 & 982 & 18.5 & 145 & 16.5 \\
\hline 4 & 956 & 18.0 & 168 & 19.1 \\
\hline 5 & 1031 & 19.5 & 223 & 25.4 \\
\hline \multicolumn{5}{|l|}{ Sleep quality in past 3 months } \\
\hline Good & 1797 & 34.0 & 293 & 33.1 \\
\hline Rather good & 1911 & 36.1 & 322 & 36.3 \\
\hline Neither good nor bad & 1101 & 20.8 & 178 & 20.1 \\
\hline Rather bad & 407 & 7.7 & 80 & 9.0 \\
\hline Bad & 73 & 1.4 & 13 & 1.5 \\
\hline \multicolumn{5}{|l|}{ Neck pain in past 3 months } \\
\hline No & 2408 & 45.1 & 393 & 44.1 \\
\hline A little bothered & 1720 & 32.2 & 289 & 32.4 \\
\hline Rather intensely bothered & 896 & 16.8 & 160 & 17.9 \\
\hline Very intensely bothered & 315 & 5.9 & 50 & 5.6 \\
\hline \multicolumn{5}{|l|}{ Low back pain in past 3 months } \\
\hline No & 2154 & 40.3 & 343 & 38.5 \\
\hline A little bothered & 1950 & 36.5 & 327 & 36.7 \\
\hline Rather intensely bothered & 887 & 16.6 & 155 & 17.4 \\
\hline Very intensely bothered & 348 & 6.5 & 67 & 7.5 \\
\hline Musculoskeletal pain (any) & & & & \\
\hline No & 608 & 11.4 & 88 & 9.9 \\
\hline A little bothered & 2036 & 38.1 & 302 & 33.9 \\
\hline Rather intensely bothered & 1722 & 32.3 & 335 & 37.6 \\
\hline Very intensely bothered & 973 & 18.2 & 167 & 18.7 \\
\hline Widespread pain & & & & \\
\hline No & 3903 & 73.1 & 658 & 73.8 \\
\hline Yes & 1436 & 26.9 & 234 & 26.2 \\
\hline
\end{tabular}

\section{Physical leisure time activities and risk of persistent fatigue at follow up}

In respondents without persistent fatigue at baseline (table 3), reported engagement in physical leisure time activities for 20 minutes or more at least once a week during the three months before baseline was associated with a reduced risk of persistent fatigue at the follow up (odds ratio $(\mathrm{OR})=0.70 ; 95 \%$ confidence interval $(\mathrm{CI}) 0.55$ to 0.89$)$, after adjustments for baseline health complaints, age, marital status, former and current smoking, tasks of a caring nature during leisure time, and work factors at baseline. The analyses of specific activities showed that reporting slow walks as the only regular activity and reporting aerobics or gymnastics were not associated, whereas reporting brisk walks and reporting other physical leisure activities were associated with the risk of persistent fatigue at follow up. 
Table 2 Respondents' physical leisure time activities during the three months before baseline by the prevalence of persistent fatigue (always or usually fatigued during daytime) at baseline

\begin{tabular}{|c|c|c|c|c|c|}
\hline \multirow[b]{3}{*}{ Physical leisure time activities } & \multirow[b]{3}{*}{$\mathbf{N}$} & \multicolumn{4}{|c|}{ Persistent fatigue at baseline } \\
\hline & & \multicolumn{2}{|l|}{ No } & \multicolumn{2}{|l|}{ Yes } \\
\hline & & $\mathbf{n}$ & $\%$ & $\mathbf{n}$ & $\%$ \\
\hline No regular exercise & 1351 & 1102 & 81.6 & 249 & 18.4 \\
\hline Slow walks* & 1006 & 837 & 83.2 & 169 & 16.8 \\
\hline Brisk walks $†$ & 1128 & 981 & 87.0 & 147 & 13.0 \\
\hline Aerobics or gymnastics $†$ & 311 & 274 & 88.1 & 37 & 11.9 \\
\hline Other activities or combinations & 1527 & 1331 & 87.2 & 196 & 12.8 \\
\hline Total sample & 5323 & 4525 & 85.0 & 798 & 15.0 \\
\hline \multicolumn{6}{|c|}{$\begin{array}{l}p \text { Value in overall } \chi^{2} \text { test }=0.000 \text {. } \\
N \text {, Total number of respondents in each category. } \\
\text { *As the only activity. } \\
\text { †As the only activity or in combination with slow walks. }\end{array}$} \\
\hline
\end{tabular}

There were only small variations in the odds ratios between the different logistic regression models (data not shown).

In respondents with persistent fatigue at baseline, there was a non-significant tendency for those who reported engagement in physical leisure time activity at least once a week to have a reduced risk of retained persistent fatigue at follow up (OR $=0.74 ; 95 \%$ CI 0.50 to 1.08$)$ after adjustments for baseline health complaints, age, marital status, former and current smoking, tasks of a caring nature during leisure time, and work factors at baseline.

\section{DISCUSSION}

In nurses' aides without persistent fatigue, reported engagement in physical leisure time activities for 20 minutes or more at least once a week was associated with a reduced risk of persistent fatigue 15 months later, also after adjustments for age, baseline health complaints, former and current smoking, marital status, tasks of a caring nature during leisure time, and work factors. The direction was the same for all activity categories. There was a non-significant tendency for those who reported engagement in physical leisure time to have reduced risk of retained persistent fatigue.

Earlier epidemiological studies also showed an association between physical inactivity during leisure time and the occurrence of persistent fatigue, ${ }^{161213}$ but most of these studies had cross sectional designs, and the one with a prospective design showed an association only in men. ${ }^{13}$ The present study is the first with a prospective design to show an association between physical leisure time activity and the risk of fatigue in women. This is also the first study to report a relation between specific leisure time activities and the risk of fatigue.

One possible explanation of the association between physical leisure time inactivity and the occurrence of persistent fatigue may be that physical activity prevents the development of persistent fatigue. Such an effect may occur through the beneficial effects that physical activity have on physical capacity, sleep quality, and mood. ${ }^{23}$ Physical activity may also have a more specific and direct effect on a person's feeling of energy. In an experimental study with repeated measures design, 10 minutes ergometer cycling improved levels of vigour, but did not have a significant effect on levels of tension, depression, or anger. ${ }^{14}$

The tendency of a reduced risk of persistent fatigue in slow walkers and in subjects engaging in aerobics or gymnastics did not reach statistical significance. Slow walking may not be strenuous enough to have preventive effects, at least not in nurses' aides. Nurses' aides usually walk a great deal at work, and slow walking during their leisure time would perhaps make no difference. Aerobics and gymnastics are strenuous activities, but there was a relatively low number of subjects engaging in these activities. Hence, low statistical power could explain the lack of statistical significance in this case.

One should bear in mind that the associations found could have been caused by background factors for which we were not able to adjust. Extensive adjustments were made for

Table 3 Relation between physical leisure time activities at baseline and prevalence of persistent fatigue (always or usually fatigued during daytime) at the 15 month follow up in respondents without persistent fatigue at baseline

\begin{tabular}{|c|c|c|c|c|}
\hline \multirow{2}{*}{$\begin{array}{l}\text { Physical leisure time activities } \\
\text { at baseline }\end{array}$} & \multirow[b]{2}{*}{$\mathbf{N}$} & \multicolumn{3}{|c|}{ Persistent fatigue at follow up } \\
\hline & & $\%(\mathbf{n})$ & OR $(95 \% \mathrm{Cl})$ & Adj OR $(95 \% \mathrm{Cl})$ \\
\hline No regular exercise & 1080 & $13.1(141)$ & 1.00 & 1.00 \\
\hline Slow walks $†$ & 815 & $11.4(93)$ & $0.86(0.65$ to 1.13$)$ & $0.80(0.58$ to 1.12$) \S$ \\
\hline Brisk walks $\ddagger$ & 968 & $9.7(94)$ & $0.72(0.54 \text { to } 0.94)^{* *}$ & $0.66(0.48 \text { to } 0.91)^{* *}$ \\
\hline Aerobics or gymnastics $\ddagger$ & 269 & $8.9(24)$ & $0.65(0.41 \text { to } 1.03)^{*}$ & $0.81(0.49 \text { to } 1.35)^{\bullet}$ \\
\hline Other regular exercise & 1315 & $8.4(111)$ & $0.61(0.47 \text { to } 0.80)^{* \star *}$ & $0.64(0.47 \text { to } 0.87)^{* \star *}$ \\
\hline Any regular exercise & 3367 & $9.6(322)$ & $0.70(0.57 \text { to } 0.87)^{* \star *}$ & $0.70(0.55 \text { to } 0.89)^{* * *}$ \\
\hline
\end{tabular}

$\mathrm{N}$, Total number of subjects in each category; $\mathrm{n}$, number of cases in each category; \%, proportion of cases in each category; OR, crude odds ratio; $95 \% \mathrm{Cl}, 95 \%$ confidence interval; Adj OR, odds ratio adjusted for age, baseline health characteristics (level of fitness, affective symptoms, sleeping problems, musculoskeletal pain, widespread pain, long term health problems of any kind), daily consumption of cigarettes, former smoking, number of preschool children, engagement with special tasks of a caring nature during leisure time, number of hours worked a week, frequency of night shifts, quantitative work demands, positive challenges in the job, the extent to which the work required physical endurance, the extent to which the culture in the work unit was supportive and encouraging, and personal engagement in the work unit, in logistic regression analysis $\left(\mathrm{N}=3971\right.$. Overall rate of correct classification $=90.1 \%$. Hosmer-Lemeshow test: $\chi^{2}=6.823 ; \mathrm{df}=$ $8 ; p=0.56$.).

Area under the ROC curve $=0.95$

${ }^{*} \mathrm{p}<0.10$; ${ }^{* *} \mathrm{p}<0.05 ;{ }^{* * *} \mathrm{p}<0.01 ; \S \mathrm{p}=0.19 ; \mathrm{q}=0.43$

†As the only activity.

†As the only activity or in combination with slow walks. 
health complaints at baseline, including the presence and severity of long term health problems of any kind, musculoskeletal pain, sleeping problems, and affective symptoms. Still, it is hard to rule out the possibility that the associations are due to differences in the baseline health situation between active and sedentary people. Other potential confounders are psychological trait factors and behavioural factors other than physical activity and smoking, such as alcohol consumption. On the other hand, the homogeneity of the cohort in sex and occupation may enhance the internal validity of this study.

The response rate in the first data collection was moderate $(62 \%)$. The list of members of the Norwegian Association of Health and Social Workers also includes retired people, and telephone contact during the collection of data gave the impression that many non-working subjects were not motivated to participate in the study. Hence, the true response rate of the vocationally active subjects was probably higher. Even so, people may have chosen not to participate in the study for reasons other than retirement or not currently working in the field. Hence, selection bias in the first sample may have influenced the results.

The number of subjects who dropped out between the first and second data collection was low (15\%), but there were some differences between the respondents and the dropouts. However, there was no difference between respondents and dropouts with respect to participation in physical leisure time activities or the prevalence of fatigue at baseline. Besides, adjustments were made for factors that differed in respondents and dropouts.

Responses to simple questions about fatigue are strongly influenced by the wording of the questions. ${ }^{24}$ Nevertheless, preliminary analyses showed that the responses to our question on fatigue were correlated with the reporting of sleep problems, affective symptoms, musculoskeletal pain, long term health problems of any kind, quantitative work demands, and tasks of a caring nature during leisure time, as one would expect (data not shown). In an earlier study of the same material, persistent fatigue predicted sickness absence, also after adjustments for other health complaints, such as affective symptoms, sleeping problems, and musculoskeletal pain. ${ }^{4}$

It may seem paradoxical that almost half of those troubled with persistent fatigue at baseline reported engagement in physical activities other than slow walks. However, the proportion of exercisers was even higher in respondents without persistent fatigue. Lack of exercise is not the only determinant of fatigue, and the high prevalence of fatigue among exercisers may be due to a wide range of factors, including psychosocial stress and physical and mental disorders. Some exercisers may have personality traits-for example, strive-achieve behaviour-that set them in stressful situations. Some patient groups may see exercise as a treatment; in another study, patients with fibromyalgia were found to have a higher level of physical leisure time activity, but lower physical fitness, than the general female population. ${ }^{25}$

The assessment of physical leisure time activities was simple, and, as for all self reporting, associated with recall bias. However, earlier studies have shown that simple self report questions can provide useful information about regular physical activity during leisure time. ${ }^{26}{ }^{27}$ In our study, physical inactivity was reported more often by older subjects, subjects with health problems, and smokers, as one would expect. ${ }^{17}$

In the composite activity category ("other activities"), less common activities were put together, even if they might differ in terms of oxygen uptake. This was unfortunate, but necessary for statistical reasons. It is unlikely that this
Take home message

Physical leisure time activity at least once a week seems to be associated with a reduced risk of developing persistent fatigue.

approach has influenced in any important way the statistical relations between the specific activity categories (slow walks, brisk walks, and aerobics or gymnastics) and the risk of fatigue. However, relations between certain activities included in the composite category and the risk of fatigue have been masked. Some of these activities, such as running, may perhaps be associated with a larger reduction in the risk of fatigue than the composite category. Other activities may perhaps have no preventive effect or may even be unhealthy.

Although people tend not to change their exercise habits, engagement in the activities may not have been stable over the 15 month observation period..$^{28}$ However, it is unlikely that changes in physical activities in the period between baseline and the 15 month follow up can explain the associations that were found. Such changes, tending to reduce the precision of the explanatory variable, would rather lead to underestimation of the true associations, and bias the estimate towards unity-for example, if some subjects with high activity at baseline stopped exercising and then developed fatigue, or if subjects with fatigue started training and this training reduced their tiredness.

In conclusion, the study supports the hypothesis that physical leisure time activities reduce the risk of developing persistent fatigue. Hence, the risk of developing persistent fatigue may be added to the list of reasons why people should be encouraged to exercise. Patients with fatigue should be asked about their physical activity, and their responses should be part of the diagnostic considerations with regard to the cause.

\section{ACKNOWLEDGEMENTS}

This study was supported financially by the Norwegian Research Council, with extra funds from the Norwegian Foundation for Health and Rehabilitation fund, The Norwegian Ministry of Health and Social Affairs, Rhône-Poulenc Rorer Ltd, Peter Møller AS, and DrTrygve Gythfeldt Research Fund.

\section{Authors' affiliations}

W Eriksen, D Bruusgaard, University of Oslo, Oslo, Norway

\section{REFERENCES}

1 Chen MK. The epidemiology of self-perceived fatigue among adults. Prev Med $1986 ; 15: 74-81$.

2 Loge JH, Ekeberg $\varnothing$, Kaasa S. Fatigue in the general Norwegian population: normative data and associations. J Psychosom Res 1998:45:53-65.

3 Nelson E, Kirk J, McHugo G, et al. Chief complaint fatigue: a longitudinal study from the patient's perspective. Fam Pract Res J 1987;6:175-88

4 Eriksen W, Bruusgaard D, Knardahl S. Work factors as predictors of sickness absence. A 3-month prospective study of nurses' aides. Occup Environ Med 2003:60:271-8.

5 David A, Pelosi A, McDonald E, et al. Tired, weak, or in need of rest: fatigue among general practice attenders. BMJ 1990;301:1199-202.

6 Stewart D, Abbey S, Meana M, et al. What makes women tired? A community sample. J Women's Health 1998;7:69-76.

7 Buchwald D, Umali P, Umali J, et al. Chronic fatigue and the chronic fatigue syndrome: prevalence in a Pacific Northwest Health Care System. Ann Intern Med 1995; 123:81-8.

8 Libbus K, Baker JL, Osgood JM, et al. Persistent fatigue in well women. Women Health 1995;23:57-72.

9 Fukuda K, Straus SE, Hickie I, et al. The chronic fatigue syndrome: a comprehensive approach to its definition and study. Ann Intern Med 1994:121:953-9.

10 Bültman U, Kant IJ, Schröer CAP, et al. The relationship between psychosocial characteristics and fatigue and psychological distress. Int Arch Occup Environ Health 2002;75:259-66. 
11 Gutstein HB. The biological basis of fatigue. Cancer 2001:92(suppl 6): 1678-83.

12 Kristal-Boneh E, Froom P, Harari G, et al. Fatigue among Israeli industrial employees. J Occup Environ Med 1996;38:1 145-50.

13 Bültman U, Kant IJ, KasI SV, et al. Lifestyle facors as risk factors for fatigue and psychological distress in the working population: prospective results from the Maastricht Cohort Study. J Occup Environ Med 2002;44:116-24.

14 Hansen CJ, Stevens LC, Coast JR. Exercise duration and mood state: how much is enough to feel better? Health Psychol 2001;20:267-75.

15 Dimeo FC, Bertz H, Finke J, et al. Aerobic exercise in the rehabilitation of cancer patients after high dose chemotherapy and autologous peripheral stem cell transplantation. Cancer 1997;79:1717-22.

16 Dimeo FC, Stieglitz R-D, Novelli-Fischer U, et al. Effects of physical activity on the fatigue and psychological status of cancer patients during chemotherapy Cancer 1999:85:2273-7.

17 Eriksen W, Bruusgaard D. Physical leisure-time activities and long-term sick leave: a 15-month prospective study of nurses' aides. J Occup Environ Med 2002;44:530-8.

18 Partinen M Gislason T. Basic Nordic Sleep Questionnaire (BNSQ): a quantitated measure of subjective sleep complaints. J Sleep Res 1995;4(suppl): 150-5.

19 Tambs K, Moum T. How well can a few questionnaire items indicate anxiety and depression? Acta Psychiatr Scand 1993;87:364-7.
20 Kuorinka I, Jonsson B, Kilbom A, et al. Standardised Nordic questionnaires for the analysis of musculoskeletal symptoms. Appl Ergon 1987;18:233-7.

21 Dallner M, Elo A-L, Gamberale F, et al. Validation of the General Nordic Questionnaire (QPSNordic) for Psychological and Social Factors at Work Nord 2000;12.

22 Hosmer DW, Lemeshow S. Applied logistic regression. 2nd ed. New York: John Wiley \& Sons, Inc, 2000

23 US Department of Health and Human Service. Physical activity and health: a report of the Surgeon General. Atlanta, GA: US Centers for Disease Control and Prevention, National Center for chronic Disease Prevention and Health Promotion, 1996.

24 Lloyd AR. Chronic fatigue and chronic fatigue syndrome: shifting boundaries and attributions. Am J Med 1998;105(suppl 3A):7S-10S.

25 Natvig B, Bruusgaard D, Eriksen W. Physical activity level and physical fitness among women with fibromyalgia. Scand J Rheumatol 1998;27:337-41.

26 Schechtman KB, Barzilai B, Rost K, et al. Measuring physical activity with a single question. Am J Public Health 1991;81:771-3.

27 Li S, Carlson E, Holm K. Validation of a single-item measure of usual physical activity. Percept Mot Skills 2000;91:593-602.

28 Matthews CE, Freedson PS, Hebert JR, et al. Seasonal variation in household, occupational, and leisure time physical activity: longitudinal analyses from the seasonal variation of blood cholesterol study. Am J Epidemiol $2001 ; 153: 172-83$ 